\title{
Potential of stem/progenitor cells in treating stroke: the missing steps in translating cell therapy from laboratory to clinic
}

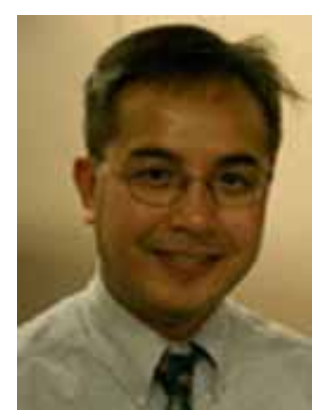

Cesar V Borlangan ${ }^{1,2 \dagger}$, Midhad Chopp?, GaryK Steinberg', Tonya M Blist, Yi Li3, Mé Lư ', DavidC Hes? \& DauglasKanctidka5

${ }^{\dagger}$ Author for correspondence ${ }^{1}$ M edical College of Georgia and Augusta VA M edical

Center, D epartment of N eurology, 1120 15th Street Augusta, GA 30904, U SA ${ }^{2}$ Tel.: +1 706721 2145; Fax: +1 7067217619 ; E-mail: cborlongan@ mail.mcg.edu ${ }^{3} \mathrm{H}$ enry Ford $\mathrm{H}$ ospital, $D$ epartment of N eurology, 2799 West Grand Blvd., Detroit, M I 48202, U SA 4Stanford U niversity School of $M$ edicine, D epartment of N eurosurgery, R281, 300 Pasteur D rive, Stanford, CA, 94305, USA ${ }^{5} U$ niversity of Pittsourgh, D epartment of N eurological Surgery, Suite B-400, U PM C, 200 Lothrop Street Pittsburgh, PA 15213, U SA
'... it is imperative for c linical

translation that these cells be tested in multiple models of focal stroke, in two species, in both gendersand in multiple la boratories.'

Accumulating laboratory evidence demonstrates the potential of transplanting stem/progenitor cells for treating CN S disorders. The realization of neuroprotection or neurorestoration in the CN S challenges the central dogma that the brain is not capable of regeneration during aging or after injury. $\mathrm{H}$ owever, despite the encouraging results that lend support to CN S regeneration, critical gating items remain that limit the translation of the laboratory findings into meaningful clinical applications of cell therapy for stroke. Foremost among the several important preclinical factors that will require additional laboratory tests and validations is the establishment of a consortium of stem cell and stroke experts who will be able to independently analyze the efficacy and safety of cell therapy in stroke.

The continued failure in drug clinical trials for treatment of acute stroke has been attributed to poor experimental designs of the drug development programs that do not adhere to the Stroke Therapy Academic Industry Round Table (STAIR) guidelines. H owever, the recent N XY-059 (StrokeAcute Ischemic N XY Treatment [SAIN T] II) trial still failed to achieve the expected efficacy despite including many of theSTAIR criteria. Accordingly, there is an urgent call to revisit the stroke drug discovery pathway [1]. There is a clear and present need to develop a consortium approach including academia, government (US FD A/NIH ) and pharmaceutical industry in drug-development programs. Equally important is recognizing the need for a translational medicine approach, in particular characterizing the drug candidate nature-target interaction, as well as determining the mechanisms underlying the phenotypic outcomes.

To this end, responding to the STAIR call, our group (Borlongan, Chopp, Steinberg, H ess and Kondziolka), together with other stroke and cellbased therapy experts, and FDA and NIH representatives, played a key role in the first Stem cell Therapeutics as an Emerging Paradigm in Stroke
(STEPS) Consortium meeting (0 ctober, 2007) whose main objective was to define several key areas that are deemed relevant to the development of cell-based, biologic and pharmacological restorative therapies (hereafter referred to as restorative therapies) for the treatment of stroke. 0 ur guiding principle was to advocate science as the catalyst for designing preclinical studies so that we can most effectively and accurately translate this research to clinical practice. This preclinical ST EPS workshop recommended the following translational criteria for designing laboratory studies on cell therapy for stroke:

- The use of appropriate species and clinically relevant type of stroke models

- The standardization of outcome measures and treatment protocols

- The need for imaging of cell tracking and host response

- The requirement for safety indices

- A call for demonstrating mechanisms of action underlying restorative therapies in ischemic stroke

To the best of our knowledge, no studies on cell therapy for stroke incorporating all these STEPS preclinical workshop recommendations exist. Despite many rodent studies showing that cell transplantation can improve recovery from stroke, the variables responsible for the success of these therapies are largely unknown. Researchers have used different cell types, rodent strains, stroke models, transplant brain target locations and time points after stroke, and behavior tests to assess the transplant's efficacy (reviewed in [2-4]). Any or all of these parameters could be critical to the outcome of functional recovery. This lack of consistency in the field makes it very difficult to compare studies and truly ascertain the optimal conditions for cell transplant therapy following stroke. For example, is good functional recovery reported in one laboratory due to the cell type used or perhaps the behavior tests employed? The realization of a collaborative effort among multiple preclinical stroke laboratories evaluating the efficacy and safety of a particular stem/progenitor cell using the same testing paradigm until now remains a challenge. In order to address this significant gap 
in our knowledge, we have assembled a consortium of three established preclinical stroke laboratories, interfaced with a data coordination center and a clinical advisory board. We recognize that the establishment of such consortium between academics and industry, with close guidance from the N IH and FD A, is the first step towards realizing this laboratory-to-clinic translational goal, resonating the core principle of both STEPS and STAIR. Accordingly, our preclinical consortium incorporates all the STEPS criteria. The primary goal is to harness a collaboration across three established preclinical stroke laboratories (Borlongan, Chopp and Steinberg) that have extensive experience in conducting laboratory studies in multiple species (rat and mouse), strain, age and gender, characterizing a variety of focal stroke models using a standardized set of behavioral and histological outcome measures, and demonstrating mechanisms of action for testing the potential of restorative therapies in ischemic stroke.

The fact that all three laboratories will carry out identical studies will help confirm the results for each parameter, which lends an unprecedented degree of veracity that is essential to a translational study. Investigation of the effect of age and gender on cell transplantation therapy is another novel aspect of our proposal as such parameters are generally overlooked in current rodent studies but are of critical clinical significance.

Our individual laboratories have performed many of the studies proposed in the ST EPS guidelines. Building on these extensive studies, we have elected to test the efficacy of two bone marrow stromal cell types (from SanBio, Inc., CA, USA, and Theradigm, Inc., M D, USA) administered at 1 month post-stroke. The 1-month time point was selected because it is clinically reasonable and desirable; neurological deficits are well defined, patients are stabilized and the extended therapeutic window permits nearly all ischemic patients to be enrolled. Two routes of cell administration, namely intracerebral (SanBio, Inc.) and intravenous (T heradigm, Inc.), will beemployed. A dosefinding analysis will be performed for both cell

types. 0 ur proposed studies are therefore designed to develop treatment paradigms that can be readily translated to clinical stroke. We also seriously considered performing imaging experiments along the line of our recently published reports $[5,6]$, which will be pursued in parallel studies. In addition, the establishment of $\mathrm{a} \mathrm{D}$ ata C oordination C enter (Lu) and a Clinical Consortium (H ess and Kondziolka) as part of our investigative team should allow us to efficiently manage and evaluate the data, and assess the clinical relevance and applicability of our preclinical studies. Lastly, our industry partnerships with SanBio, Inc. and Theradigm, Inc. facilitates an ample supply of transplantable cells produced under good manufacturing practice, quality assurance and quality control regimen, which should expedite our translational goal of delivering a clinical grade product to the clinic. In summary, our preclinical STEPS consortium is desirous to deliver missing critical steps in the translational testing and validation of efficacy and safety of cell therapy in ischemic stroke, which, to date, has not been realized in the field of neurorestoration. Although these cell treatments have demonstrated therapeutic efficacy in individual laboratories [2-8], it is imperative for clinical translation that these cells be tested in multiple models of focal stroke, in two species, in both genders and in multiple laboratories.

Financial \& competing interests disclosure CV Borlongan and DC Hess are funded by $\mathrm{NIH}$ grants 1 U 01 NS055914 and 2R42 NS055606; CV Borlongan and $D$ Kondziolka are Consultants to SanBio, InC; M Chopp is funded by NIH grants PO1 NS42345 and P50 N 223393; GK Steinberg is funded by N IH N IN DS grants RO 1 N S27292 and P01 N S37520, and is a stockholder in Cardium Therapeutics and has stock options in Stem Cells, Inc., and N eurofluidics. The authors have no other relevant affiliations or financial involvement with any organization or entity with a financial interest in or financial conflict with the subject matter or materials discussed in the manuscript apart from those di sclosed.

$\mathrm{N}$ o writing assistance was utilized in the production of this manuscript.

Bibliography

1. Feuerstein $\mathrm{GZ}$, Zaleska M M , K rams M et al.: $M$ issing steps in the STAIR case: a translational medicine perspective on the development of N XY-059 for treatment of acute ischemic stroke. J. Cereb. Blood Flow M etab. 28, 217-219 (2008).

2. Chopp M, Li Y: Treatment of neural injury with marrow stromal cells. Lancet N eurol. 1, 92-100 (2002).
3. Bliss T, Guzman R, D aadi M , Steinberg GK: C ell transplantation therapy for stroke. Stroke 38, 817-826 (2007).

4. Borlongan CV, Fournier C, Stahl CE et al.: Gene therapy, cell transplantation and stroke. Front Biosci. 11, 1090-1101 (2006).

5. Chopp M, Zhang ZG, Jiang Q : $N$ eurogenesis, angiogenesis, and M RI indices of functional recovery from stroke. Stroke 38, 827-831 (2007).
6. Guzman R, U chida N, BlissTM et al.: Long-term monitoring of transplanted human neural stem cells in developmental and pathological contexts with M RI. Proc. Natl Acad. Sci. U SA 104, 10211-10216 (2007).

7. H ess D C, Borlongan CV : Stem cells and neurological diseases. Cell Prolif. 41, 94-114 (2008).

8. Kondziolka D, Wechsler $L$, Achim C: N eural transplantation for stroke. J. Clin. N eurosci. 9, 225-230 (2002). 